\title{
Commentary: The quest for the holy grail continues: Is levosimendan the best choice to support patients with cardiomyopathy requiring cardiac surgery?
}

\author{
Francesco Onorati, MD, PhD, ${ }^{\mathrm{a}}$ Gianfranco Veraldi, $\mathrm{MD},{ }^{\mathrm{b}}$ and Giuseppe Faggian, $\mathrm{MD}^{\mathrm{a}}$
}

\author{
From the ${ }^{\mathrm{a}}$ Division of Cardiac Surgery, University of Verona Medical School; and ${ }^{\mathrm{b}}$ Vascular Surgery Unit, \\ University Hospitals in Verona, Verona, Italy. \\ Disclosures: Authors have nothing to disclose with regard to commercial support. \\ Received for publication June 26, 2019; revisions received June 26, 2019; accepted for publication June 28, 2019; \\ available ahead of print Oct 22, 2019. \\ Address for reprints: Francesco Onorati, MD, PhD, Division of Cardiac Surgery AOUI Verona; Piazzale Stefani \\ n.1, 37126, Verona, Italy (E-mail: francesco.onorati@univr.it). \\ J Thorac Cardiovasc Surg 2020;159:2312-3 \\ $0022-5223 / \$ 36.00$ \\ Copyright $(c) 2019$ by The American Association for Thoracic Surgery \\ https://doi.org/10.1016/j.jtcvs.2019.06.136
}

In this subanalysis of the multicenter, randomized, phase 3 LEVO-CTS (Levosimendan in Patients With Left Ventricular Systolic Dysfunction Undergoing Cardiac Surgery Requiring Cardiopulmonary Bypass) trial, the authors demonstrated a beneficial effect of levosimendan in patients with reduced ejection fraction undergoing isolated coronary artery bypass grafting (ie, with ischemic cardiomyopathy requiring surgical revascularization) in terms of cardiac output, episodes of low cardiac output syndrome, and requirement of prolonged inotropic support. ${ }^{1}$ They also highlighted that patients receiving perioperative levosimendan support have a long-lasting benefit in terms of 30-day and 90-day all-cause mortality. Finally, a mean difference in acute care costs of \$2419 lower with levosimendan than placebo was reported in ischemic patients (although this net-gain was lost when costs of levosimendan was considered). Unfortunately, these data were not confirmed in patients undergoing isolated valve surgery (where a paradoxical detrimental effect of levosimendan seems to emerge), as well as in patients undergoing combined procedures. ${ }^{1}$

The beneficial role of levosimendan in ischemic cardiomyopathy dates back to several single-center trials performed during the early $2000 \mathrm{~s}^{2}$ : indeed, these results were questioned by a recent meta-analysis of Lee and colleagues $^{3}$ that considered studies in patients with moderate-to-severe left ventricular dysfunction, as well as by the 3 most recent multicenter randomized trials. ${ }^{4-6}$ Indeed, the LEVO-CTS trial failed to demonstrate any advantage in levosimendan-supported patients with left ventricular ejection fraction $\leq 35 \%{ }^{4}$; the LICORN (Levosimendan in Coronary Artery Revascularization) trial did not find any advantage in patients with coronary artery disease and left ventricular ejection fraction $\leq 40 \%$ in a primary composite endpoint reflecting low cardiac output syndrome $^{5}$; the CHEETAH (Levosimendan to Reduce

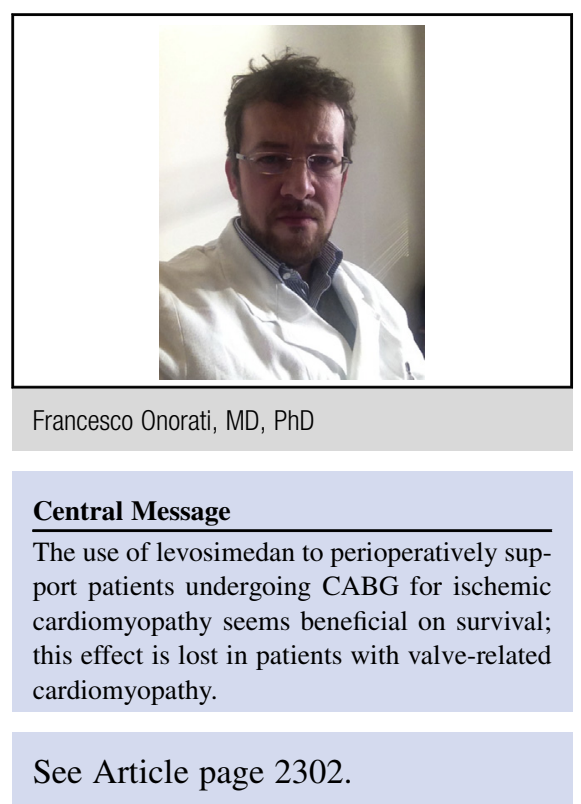

Mortality in High-Risk Cardiac Surgery Patients: A Multicenter Randomized Controlled Trial) trial was prematurely stopped for futility because of the absence of any between-group difference in survival, other efficacy endpoints, and adverse events in patients developing postoperative left ventricular dysfunction. ${ }^{6}$

All studies published on the topic have consistent limitations deserving balanced interpretations of the results. ${ }^{2}$ Similarly, the paper published in this issue of the Journal should be interpreted with caution. First, the study is underpowered to evaluate the safety and efficacy of this drug in the subgroups of patients considered for this analysis. Second, it is quite difficult to understand-from a physiopathology point of view-how a subtle difference in cardiac index after the start of a drug might translate in a survival benefit lasting at 90 days after surgery, especially in light of a comparable incidence of rough events and complications (perioperative acute myocardial infarction, need for mechanical assisting device, or renal-replacement therapies). That is of utmost importance, given also that nothing is known about viable myocardium in levosimendan- or placebo-treated populations. Third, despite the fact that the original LEVO-CTS trial was randomized, this subanalysis is not, and small differences in baseline prognostic factors (diabetes mellitus, heart 
failure, New York Heart Association class III/IV) in the ischemic population might have combined to lead to apparent (but inexistent) treatment differences.

Despite these limitations, however, punctually addressed by the authors in the paper, this study is important because it contradicts "back" the results of the aforementioned randomized controlled trials. In particular, this subanalysis clearly points the finger at a differential effect of this drug in different surgical scenarios, committing researchers to investigate further the role of levosimedan in specific subsets of patients receiving different interventions. The quest for the holy grail is just started.

\section{References}

1. van Diepen S, Mehta RH, Leimberger JD, Goodman SG, Fremes S, Jankowich R, et al. Levosimendan in patients with reduced left ventricular function undergoing isolated coronary or valve surgery. J Thorac Cardiovasc Surg. 2020;159: 2302-9.e6.

2. Guarracino F, Heringlake M, Cholley B, Bettex D, Bouchez S, Lomivorotov VV et al. Use of levosimendan in cardiac surgery: an update after LEVO-CTS, CHEETAH, and LICORN trials in the light of clinical practice. $J$ Cardiovasc Pharmacol. 2018;71:1-9.

3. Lee CT, Lin YC, Yeh YC, Chen TA, Chen CY. Effects of levosimendan for perioperative cardiovascular dysfunction in patients receiving cardiac surgery: a meta-analysis with trial sequential analysis. Intensive Care Med. 2017;43: 1929-30.

4. Mehta RH, Leimberger JD, van Diepen S, Meza J, Wang A, Jankowich R, et al Levosimendan in patients with left ventricular dysfunction undergoing cardiac surgery. N Engl J Med. 2017;376:2032-42.

5. Cholley B, Caruba T, Grosjean S, Amour J, Outtara A, Villacorta J, et al Effect of levosimendan on low cardiac output syndrome in patients with low ejection fraction undergoing coronary artery bypass grafting with cardiopulmonary bypass: the LICORN randomized clinical trial. JAMA. 2017;318:548-56.

6. Landoni G, Lomivorotov VV, Alvaro G, Lobreglio R, Pisano A, Guarracino F, et al. Levosimendan for hemodynamic support after cardiac surgery. $N$ Engl J Med. 2017;376:2021-31. 